\title{
Validity of Driving Simulator for Agent-Human Interaction
}

\author{
Yutao Ba, Wei Zhang, and Gavriel Salvendy \\ Department of Industrial Engineering, Tsinghua University, Beijing, 100084, China \\ bb0438@163.com, zhangwei@tsinghua.edu.cn, salvendy@purdue.edu
}

\begin{abstract}
Drivers of intelligent agent in the virtual environment offer researchers a great opportunity to study interaction between drivers in the safe, controlled, replicable and low-cost simulator environment. However, the validation for the effectiveness of agent drivers upon human drivers is required. The present study aimed to evaluate the validity of agent-human interaction in a simulator, compared with human-human interaction on real roads. 20 male participants were recruited to watch eight scenarios concerning interactive driving behaviors and signal usage, which were presented in the forms of both realistic films with human drivers and virtual graphic scenes with agent drivers. Participants' attitude, emotions and visual attention were recorded. The relative validity was established for all measurements. This result suggested that medium fidelity simulator with agent drivers could provide the effective values to evaluate the human-human interaction mirrored these values obtained on real road.
\end{abstract}

Keywords: validity, intelligent agent, driving simulator, vehicle signals.

\section{Introduction}

\subsection{Agent-Human Interaction in Driving Simulator}

From the last decade, intelligent agents with autonomous behaviors have received large amounts of interest in various research areas (Bonabeau 2002). Concerning driving safety research, agent-based drivers in the virtual environment could simulate the driving behaviors (e.g. following, merging or overtaking) as human drivers conduct on the real roads (Ehlert and Rothkrantz 2001, Bonabeau 2002, Doniec et al. 2008). This advantage provides researchers a great opportunity to study the effect of other vehicles on the driver's visual perception, cognitive process and according behaviors in the safe, controlled, replicable and low-cost simulator environment. However, a key component of requirements for such agent-human interaction study is the validation for the effect of agents upon human (Hudlicka 2003). In other words, does a driver feel real while interacting with agent drivers in the specific virtual scenarios, in comparing with his/her daily driving surrounded by other human drivers? 


\subsection{Validity of Driving Simulator}

The previous validation studies concerning driving simulators proposed two levels of validity: physical validity and behavioral validity (Blaauw 1982, Yan et al. 2008, Wang et al. 2010). Physical validity refers to the physical correspondence of the simulator's components, layout, and dynamics with its real world counterpart. Therefore, a moving-base, high-fidelity driving simulator was often assumed to have greater physical validity than a fixed-base, low-fidelity simulator. Behavioral validity refers to the consistency of drivers' responses in simulator and it on real roads. No level of physical validity is useful to human factors research if behavioral validity cannot be established. Absolute behavioral validity is established when the numerical values in simulator and on road are basically the same. Relative behavioral validity requires that the effects of controlled factors are of the same direction and with similar magnitude (or significance) in the two test environments. The relative behavioral validity is the minimum requirement for simulator to reflect the actual response in realistic driving.

As a part of research of driver-driver interaction (Ba and Zhang 2012, Ba et al. 2013), the primary purpose of the present study was to validate the agent-human interaction in specific driving scenarios with communicational signals (e.g. hazard lights, turn signals and horn). The drivers' attitude, emotions and visual attention were measured to evaluate the effects of signals on drivers' cognitive states and visual perception. To compare the difference of these effects in stimulator and on the real road, two types of stimuli were presented: realistic films with human drivers and virtual graphic scenes with agent drivers.

\section{Methodology}

\subsection{Participants}

Twenty male drivers (from 20 to 29 years old) were recruited from a university population thorough campus Online Bulletin Board. All participants were required to have a minimum of three years of active driving experience and more than 20,000 kilometers' total driving distance. Participants were required to sign an institutionally approved informed consent form before experiment and were provided with RMB 100 Yuan (about 15 U.S. dollars) after experiment.

\subsection{Film of Human Drivers and Medium Fidelity Graphic Scene of Agent Drivers}

Consistent with the task protocol proposed in previous study (Ba et al. 2013), this experiment contained eight common scenarios that subject interacted with another vehicle, labeled as signaling vehicle: 1) signaling vehicle in front is parked (Hazard Lights); 2) signaling vehicle in front is traveling at low speed (Hazard Lights); 3) signaling vehicle in front is staring and intending to enter the subject's lane (Turn Signal); 4) signaling vehicle in front is stopping into the park lane (Turn Signal); 
5) signaling vehicle behind is moving left to overtake subject's vehicle (Turn Signal); 6) signaling vehicle is merging from left lane (Turn Signal); 7) Signaling vehicle is overtaking after subject's vehicle yield the way (Horn); 8) Signaling vehicle is requesting subject vehicle to move out of the way (Horn).

Each scenario included two paired scenes, signal-use scene and none-signal scene. In none-signal scene, signaling vehicle conducted the same behaviors as it did in the paired signal-use scenes, e.g. signaling vehicle was parked with hazards lights in signal-use scene versus signaling vehicle was parked without any signal in nonesignal scene. To validate the agent-human interaction in simulator, each scene was presented in two types of stimuli, realistic film with human driver and medium fidelity graph with agent driver (Fig. 1). Thus, eight scenarios totally included 32 scenes ( 8 human signal-use, 8 agent signal-use, 8 human non-signal and 8 agent nonsignal).

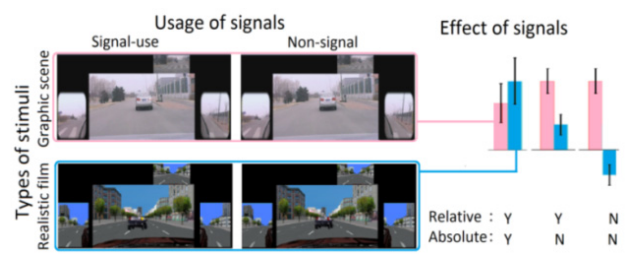

Fig. 1. Illustration of independent variables and establishment of validity

\subsection{Measurements}

Participant's attitude towards the signaling vehicle was measured by a validated fivepoint scale based on the Theory of Planned Behavior. A five-point version Self Assessment Manikin was used to measure drivers' emotion states, which included three independent dimensions: pleasure, arousal and dominance. Participants' visual attention towards the signaling vehicle was measured via a SMI IVIEM XTM headmounted gaze tracker. The fixation was defined as the duration (longer than $80 \mathrm{~ms}$ ) of continuous gaze points on the signaling vehicle.

\section{$2.4 \quad$ Procedure}

During the experiment, participant was settled in the driver's seat of a fixed-base simulator. Eye tracker was then calibrated following the manufacturer's instructions. All 32 scenes were presented in the flat screen in front of simulator $(3.2 \times 2.5 \mathrm{~m}, 3 \mathrm{~m}$ from driver's seat) with different random sequences for each participant. During watching, participants' fixations were recorded by eye tracking system. After each scene, there was a short break to let participant rate attitude and emotion. 


\section{$3 \quad$ Analysis and Results}

\subsection{Statistic Methods}

Two independent variables were signals (signal-use vs. non-signal) and stimuli types. Dependent variables included attitude, emotion states (pleasure, arousal and dominance) and visual attention (total fixation time, fixation duration and fixation frequency). Repeated-measure General Linear Model was applied to test the validity of agent drivers' effect in simulator. When the main effect of signals was in the same direction with significance for both stimuli types, the relative validity was established. When relative validity was established and no significant main effect of stimuli types was reported, the absolute validity established (illustrated in Fig. 1). The results for all independent variables of eight scenarios are showed in Table 2.

\subsection{Cognitive states - Attitude and Emotion}

The main effects of signals on attitude was consistent between two stimuli types in all scenarios, significantly increased attitude in scenario 1-7 and significantly decreased attitude in scenario 8. Meanwhile, no significant difference was reported between two stimuli types. Thus absolute validity was established for the effect of agent drivers' signals on the attitude. The significant effect of signals was also demonstrated for pleasure and arousal in all scenarios and the direction of effect was consistent for both stimuli types. Signals significantly increased pleasure in scenario 1-7 and significantly decrease attitude in scenario 8. Arousal was increased in all scenarios. However, the significant main effect of stimuli types was reported in scenarios 4, 5, 6 and 7 for pleasure, and in scenario 3, 4, 5, 6 and 7 for arousal. Thus, only relative validity established for the effect of agent drivers' signals on pleasure and arousal. As to the emotional dimension of dominance, the main effect of signals was not statistically distinguishable in scenario $1,2,3,7$ and 8 . The definition of validity could be not suitable for these cases. Therefore, we only analyzed the validity in scenario 5 and 6 . The signals in realistic films significantly decreased the dominance in scenario 5 and 6. The notable reduction also reported for graphic stimuli. Because of the main effect of stimuli types, only relative validity was established for the effect of agent drivers' signals on dominance.

Table 1. Significance of main effects and validity

\begin{tabular}{|c|c|c|c|c|c|c|c|}
\hline \multirow{2}{*}{ Scenarios } & \multicolumn{7}{|c|}{ Effects of signals in realistic films $\left(\mathrm{F}_{1,19}\right)$} \\
\hline & AT & PL & $\mathrm{AR}$ & DO & FT & FD & $\mathrm{FF}$ \\
\hline 1 & $35.35 * *$ & $14.65 * *$ & $21.53 * *$ & 2.51 & $18.31 * *$ & $10.85 * *$ & 0.37 \\
\hline 2 & $63.38 * *$ & $36.65 * *$ & $7.86 *$ & 1.18 & $35.11 * *$ & $14.78^{* *}$ & 1.05 \\
\hline 3 & $68.26 * *$ & $57.28 * *$ & $5.54 *$ & 2.88 & $44.87 * *$ & $19.55 * *$ & 1.12 \\
\hline 4 & $24.56 * *$ & $46.54 * *$ & $4.65 *$ & 3.61 & $48.98 * *$ & $8.15^{*}$ & 0.89 \\
\hline 5 & $53.24 * *$ & $37.16 * *$ & $6.07 *$ & $6.89 *$ & $33.29 * *$ & $23.51 * *$ & $6.39 *$ \\
\hline 6 & $61.54 * *$ & $48.55^{* *}$ & $4.55^{*}$ & $11.60 * *$ & $8.93 * *$ & $9.55^{* *}$ & $8.19 *$ \\
\hline 7 & $16.87 * *$ & $11.23 * *$ & $23.11 * *$ & 2.55 & $17.54 * *$ & $19.55^{* *}$ & $8.14 *$ \\
\hline 8 & $9.53 * *$ & $8.99 * *$ & $16.53 *$ & 3.59 & $8.03 *$ & $4.65^{*}$ & $11.05 * *$ \\
\hline
\end{tabular}


Table 1. (continued)

\begin{tabular}{|c|c|c|c|c|c|c|c|}
\hline & \multicolumn{7}{|c|}{ Effects of signals in graphic scenes $\left(\mathrm{F}_{1,19}\right)$} \\
\hline 1 & $48.45^{* *}$ & $16.78 * *$ & $18.48 * *$ & 1.15 & $16.53 * *$ & $12.55^{* *}$ & 0.56 \\
\hline 2 & $71.21 * *$ & $34.12 * *$ & $7.51^{* *}$ & 3.14 & $6.14 *$ & $11.23^{* *}$ & 0.08 \\
\hline 3 & $74.55^{* *}$ & $81.55^{* *}$ & $5.65 * *$ & 3.58 & $5.51 *$ & $16.52^{* *}$ & 0.32 \\
\hline 4 & $53.21 * *$ & $31.94 * *$ & $6.77 *$ & $4.89^{*}$ & $4.43^{*}$ & $9.15^{* *}$ & 0.12 \\
\hline 5 & $61.81 * *$ & $45.94 * *$ & $6.68^{*}$ & $7.11 *$ & $5.14 *$ & $11.25^{* *}$ & $4.34 *$ \\
\hline 6 & $71.58^{* *}$ & $39.65 * *$ & $7.74 *$ & $6.68^{*}$ & $9.55^{* *}$ & $13.54 *$ & $5.56^{*}$ \\
\hline 7 & $18.55^{* *}$ & $18.15^{* *}$ & $11.15^{*}$ & 3.11 & $69.66^{* *}$ & $48.21 *$ & $3.48^{*}$ \\
\hline \multirow[t]{2}{*}{$\underline{8}$} & $9.87 * *$ & $8.18 * *$ & $13.58^{*}$ & 0.85 & $11.59 * *$ & $17.56^{*}$ & $5.52 *$ \\
\hline & \multicolumn{7}{|c|}{ Effect of stimuli types $\left(\mathrm{F}_{1,19}\right)$} \\
\hline 1 & 2.67 & 0.42 & 1.91 & 2.88 & $15.85^{* *}$ & 2.53 & 0.12 \\
\hline 2 & 1.13 & 0.04 & 0.59 & $6.89 *$ & $25.13^{* *}$ & 0.72 & $4.89 *$ \\
\hline 3 & 2.63 & 0.89 & $6.38 *$ & $8.04 *$ & $43.65^{* *}$ & $4.15^{*}$ & $9.15^{* *}$ \\
\hline 4 & 1.85 & $3.74 *$ & $5.81^{*}$ & 2.54 & $38.77^{* *}$ & 2.17 & $6.75^{*}$ \\
\hline 5 & 3.42 & $7.16^{*}$ & $6.29 *$ & $5.45^{*}$ & $38.12 * *$ & 0.21 & $8.95^{* *}$ \\
\hline 6 & 2.32 & $6.45^{*}$ & $5.95^{*}$ & $6.55^{*}$ & $5.57 *$ & 0.84 & 1.26 \\
\hline 7 & 1.14 & $7.86^{*}$ & $4.48^{*}$ & $3.31 *$ & $45.55^{* *}$ & $9.54 * *$ & 1.35 \\
\hline \multirow[t]{2}{*}{8} & 0.77 & 1.55 & 3.21 & 2.55 & $4.15^{*}$ & 0.35 & $9.68 * *$ \\
\hline & \multicolumn{7}{|c|}{ Validity(Relative/Absolute) } \\
\hline 1 & $\mathrm{Y} / \mathrm{Y}$ & $\mathrm{Y} / \mathrm{Y}$ & $\mathrm{Y} / \mathrm{Y}$ & - & $\mathrm{Y} / \mathrm{N}$ & $\mathrm{Y} / \mathrm{Y}$ & - \\
\hline 2 & $\mathrm{Y} / \mathrm{Y}$ & $\mathrm{Y} / \mathrm{Y}$ & $\mathrm{Y} / \mathrm{Y}$ & - & $\mathrm{Y} / \mathrm{N}$ & $\mathrm{Y} / \mathrm{Y}$ & - \\
\hline 3 & $\mathrm{Y} / \mathrm{Y}$ & $\mathrm{Y} / \mathrm{Y}$ & $\mathrm{Y} / \mathrm{N}$ & - & $\mathrm{Y} / \mathrm{N}$ & $\mathrm{Y} / \mathrm{N}$ & - \\
\hline 4 & $\mathrm{Y} / \mathrm{Y}$ & $\mathrm{Y} / \mathrm{N}$ & $\mathrm{Y} / \mathrm{N}$ & - & $\mathrm{Y} / \mathrm{N}$ & $\mathrm{Y} / \mathrm{Y}$ & - \\
\hline 5 & $\mathrm{Y} / \mathrm{Y}$ & $\mathrm{Y} / \mathrm{N}$ & $\mathrm{Y} / \mathrm{N}$ & $\mathrm{Y} / \mathrm{N}$ & $\mathrm{Y} / \mathrm{N}$ & $\mathrm{Y} / \mathrm{Y}$ & $\mathrm{Y} / \mathrm{N}$ \\
\hline 6 & $\mathrm{Y} / \mathrm{Y}$ & $\mathrm{Y} / \mathrm{N}$ & $\mathrm{Y} / \mathrm{N}$ & $\mathrm{Y} / \mathrm{N}$ & $\mathrm{Y} / \mathrm{N}$ & $\mathrm{Y} / \mathrm{Y}$ & $\mathrm{Y} / \mathrm{Y}$ \\
\hline 7 & $\mathrm{Y} / \mathrm{Y}$ & $\mathrm{Y} / \mathrm{N}$ & $\mathrm{Y} / \mathrm{N}$ & - & $\mathrm{Y} / \mathrm{N}$ & $\mathrm{Y} / \mathrm{N}$ & $\mathrm{Y} / \mathrm{Y}$ \\
\hline 8 & $\mathrm{Y} / \mathrm{Y}$ & $\mathrm{Y} / \mathrm{Y}$ & $\mathrm{Y} / \mathrm{Y}$ & - & $\mathrm{Y} / \mathrm{N}$ & $\mathrm{Y} / \mathrm{Y}$ & $\mathrm{Y} / \mathrm{N}$ \\
\hline
\end{tabular}

AT-attitude, PL-pleasure, AR-arousal, DO-dominance, FT-total fixation time, FD-fixation duration, FF-fixation frequency. $* \mathrm{p}<0.05, * * \mathrm{p}<0.01$

\subsection{Visual Attention- Fixation Time, Fixation duration and Fixation Frequency}

The signals of both stimuli types significantly increased participants' total fixation time towards the signaling vehicle in all scenarios. However, the significant difference was reported between two stimuli types in all scenarios. Thus the relative validity was obtained for the effect of agent drivers' signals on the total fixation time. As to the fixation duration, the signals of both stimuli types significantly increased participants' fixation duration in all scenarios. The significant main effect of stimuli types was obtained in scenario 3 and 7 . This indicated that only relative validity established for upon fixation duration. When considering the fixation frequency, it should be emphasized that the significant difference between non-signal scenes and signal-use scenes only established in scenario 5, 6, 7 and 8, where the signaling vehicles appeared in the region out of the road ahead. Among these scenarios, significant difference between two stimuli types was demonstrated in scenario 5 and 8. Thus, we only achieved the relative validity for the effect of agent drivers' signals on the fixation frequency. 


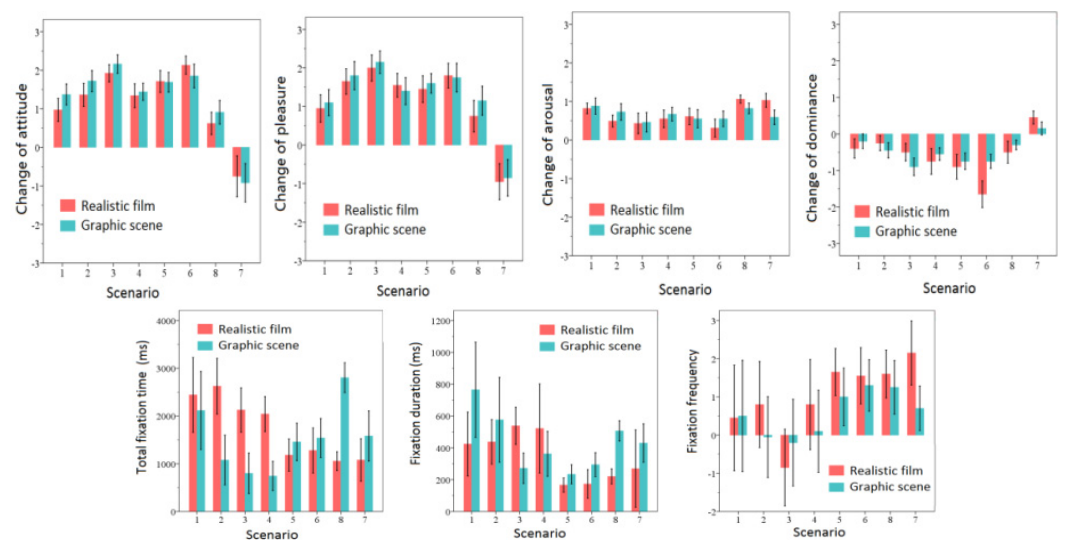

Fig. 2. Effect of signals upon independent variables (Mean \pm SD) across two stimuli types

\section{Discussion and Conclusion}

In summary, the relative validity was obtained for all of selected measurements. As to the variable of attitude, we can even achieve the absolute validity for the graphic stimuli of agent drivers. Although the significant difference between two proposed stimuli types existed for the variables of emotional states and visual attention. These gaps did not harm the change tendency of these variables induced by signals. Therefore, agent-human interaction with signals in driving simulator could produce the validated effects on participants' cognitive state and visual perception as it in the situation of human-human interaction on real roads.

\section{Reference}

1. Ba, Y., Zhang, W.: An empirical approach for driver-driver interaction study: Attributes, influence factors and framework. Advances in Usability Evaluation, 404 (2012)

2. Ba, Y., Zhang, W., Yang, Y., Salvendy, G.: Interpersonal signal processing during interactive driving scenarios. Working paper (2013)

3. Blaauw, G.J.: Driving experience and task demands in simulator and instrumented car: A validation study. Human Factors: The Journal of the Human Factors and Ergonomics Society 24(4), 473-486 (1982)

4. Bonabeau, E.: Agent-based modeling: Methods and techniques for simulating human systems. Proceedings of the National Academy of Sciences of the United States of America 99(3), 7280-7287 (2002)

5. Doniec, A., Mandiau, R., Piechowiak, S., Espié, S.: A behavioral multi-agent model for road traffic simulation. Engineering Applications of Artificial Intelligence 21(8), 14431454 (2008)

6. Ehlert, P.A., Rothkrantz, L.J.: Microscopic traffic simulation with reactive driving agents. In: Proceedings of the Intelligent Transportation Systems, pp. 860-865. IEEE (2001) 
7. Hudlicka, E.: To feel or not to feel: The role of affect in human-computer interaction. International Journal of Human-Computer Studies 59(1), 1-32 (2003)

8. Wang, Y., Mehler, B., Reimer, B., Lammers, V., D’Ambrosio, L.A., Coughlin, J.F.: The validity of driving simulation for assessing differences between in-vehicle informational interfaces: A comparison with field testing. Ergonomics 53(3), 404-420 (2010)

9. Yan, X., Abdel-Aty, M., Radwan, E., Wang, X., Chilakapati, P.: Validating a driving simulator using surrogate safety measures. Accident Analysis \& Prevention 40(1), 274 $288(2008)$ 